\title{
Load Growth and Power Flow Control with DSRs: Balanced vs Unbalanced Transmission Networks
}

\author{
Shaimaa Omran ${ }^{\mathrm{a},{ }^{*}}$, Robert Broadwater ${ }^{\mathrm{b}}$, Joshua Hambrick ${ }^{\mathrm{c}}$, Murat Dilek ${ }^{\mathrm{c}}$, ChifongThomas ${ }^{\mathrm{d}}$, and Frank \\ Kreikebaum $^{\text {d }}$ \\ a Engineering Research Division, National Research Centre, Cairo 12622 Egypt \\ ${ }^{\mathrm{b}}$ ECE department, Virginia Tech, Blacksburg, VA 24061 USA \\ ${ }^{\mathrm{c}}$ Electrical Distribution Design, Blacksburg, VA 24061 USA \\ d Smart Wire Grid, Oakland, CA, 94612 USA
}

Abstract: An important issue in today's power system is the need to analyze and determine the adequacy of transmission capacity. There is a need for approaches to increase transmission system capacity without construction of new transmission facilities, all while assuring secure operation of the grid. Distributed Series Reactors (DSRs) are a new smart grid technology that can be applied to control flows in transmission or distribution systems. DSRs can be used to balance phase flows in a single line as well as to control the distribution of flow in a meshed system. This paper investigates DSRs to control power flow to alleviate overloads due to increased power transfer. The IEEE 39 bus standard model is modified to a 3-phase unbalanced transmission model with $345 \mathrm{kV}$ lines that accounts for tower geometry. Using the symmetrical components transformation, a balanced, 3-phase model is then derived from the unbalanced, 3-phase model. DSR designs based on the unbalanced, 3-phase model and the balanced, 3-phase model are compared and used to demonstrate the effectiveness of DSR control in handling load growth. Only unbalanced impedances are addressed i.e., non-transposed lines, but the effects of impedance unbalance are shown to be significant on the resulting DSR design.

Keywords: Distributed Series Reactor (DSR) Design, Electric Power Transfer, Positive Sequence Impedance Model, Power Flow Control, Balanced/Unbalanced Transmission Systems.

\section{Introduction}

Despite the issuance of U.S. Energy Policy Act of 2005 "EPAct 2005", intended to attract investment in the transmission grid, the risks associated with planning, siting, permitting, and constructing new transmission facilities are considered a major challenge. This was also recognized by the Policy Statement that FERC released recently stating that developing

\footnotetext{
${ }^{*}$ Corresponding author. Tel.: +201067813566.

Email addresses: shmomran@vt.edu (S Omran), dew@vt.edu (R Broadwater), josh-hambrick@edd-us.com(JHambrick), murat-dilek@eddus.com (M Dilek), Chifong.Thomas@smartwiregrid.com (C Thomas), Frank.Kreikebaum@ smartwiregrid.com (F Kreikebaum)
} 
transmission presents risks and challenges unlike investment in any other utility plant [1]. Utilities in North America and all over the world are trying to incorporate new technological innovations to expand the transmission capacity using current assets and facilities [2]. Ways to enhance transmission system capacity with no new transmission construction have been presented in the literature [3]-[6]. In [3] and [4] the authors discuss series and shunt compensation to enable better utilization of generation and transmission facilities and enhance the transmission capacity. Reference [5] suggests an increase of transmission capacity by symmetrisation of sound phases after a 1-phase fault occurrence. Reference [6] investigates the thermal behaviour of overhead lines to provide higher utilisation of the transmission lines.

Controlling power flow over lines to enhance system capacity, alleviate overloads, and improve reliability has also been addressed in [7]-[17]. Reference [7] proposes a multi-agent system for coordinated and adaptive control of power flow over transmission lines to relieve overloads. References [8]-[10] investigate using Flexible AC Transmission System (FACTS) devices for power flow control. Reference [11] illustrates three generic types of FACTS devices for power flow control.

A new smart grid technology, the Distributed Series Reactor (DSRs), is investigated in this paper. DSRs can be used to balance flows in the phases of an unbalanced line, or used to control the distribution of flow in a meshed network. In this paper, DSRs will be used to alleviate overloads that result due to increased load. The design is performed for an unbalanced, 3-phase system, and then for a balanced, 3-phase model derived from the unbalanced model, where the symmetrical components transformation is used to create the balanced model. 
Reference [12] proposed the use of an active smart wire, which is a DSR module with an AC capacitor, two AC switches and a communications package, for controlling power flows on a strained grid. In [13] the authors studied the impact of smart wires, which are standard transmission lines that are augmented with DSRs, in reduction of transmission investment required to implement policies like the Renewable Portfolio Standards (RPS). References [14] and [15] introduced the concept of Distributed FACTS (D-FACTS) as an alternative approach to realizing cost-effective power flow control. In [14] the distributed static series compensator (DSSC) device was introduced as a means to control power flow in transmission systems. Reference [15] discusses the concept of distributed series impedance and distributed static series compensators.

In [16] the authors investigated the DSR system design over parallel lines by performing a series of experiments using three long parallel transmission lines. Several case studies were evaluated in which the three long lines were variously modeled to investigate the impact of different line models (lumped vs. distributed), and impedance models (balanced vs. unbalanced). Deployment of DSRs over one of the three long transmission lines improved the utilization of the capacity of the parallel long lines and increased the maximum power delivered to the load. In this paper DSR system design is performed for a meshed network with lines of different lengths. Here the designs are compared for an unbalanced, 3-phase model and a balanced, 3phase model, where the balanced model is derived from the unbalanced model. Of interest are errors that may arise with the balanced assumption.

It is worth noting that the lines of the physically modelled IEEE 39 bus system are modelled in this paper as lumped $\pi$-models, where line lengths ranged from 20 miles to 100 miles. Lumped $\pi$-models provide equivalent results to distributed line models when the line lengths are less than 
100 miles. However, as lines become very long, such as 200 miles, the lumped $\pi$-model underestimates the number of DSRs needed for power flow control. Furthermore, the placement of the DSRs on the long lines, such as at the sending end bus or receiving end bus, has different effects on the power flows [16].

Design of DSRs to control power flow over transmission lines to alleviate overloads due to load growth under single line contingencies is investigated in [17]. N-1 contingency analysis is performed to assure secure operation of the grid while controlling the active power flow.

No previous papers in the literature other than the work in [16] and [17] studied the application of DSRs in unbalanced three phase transmission systems, and many of the transmission lines in the US are unbalanced [18-21]. This work is devoted to investigating the application of DSRs in unbalanced transmission for load growth.

\section{DSR Technology}

For the sake of reliability, the existing transmission grid is designed as a meshed network. In a transmission system, one of the lines may become overloaded while a line along an alternative path may be lightly loaded. Rerouting the flow from overloaded lines to more lightly loaded lines can improve the utilization of the existing transmission system, thus delaying the investment in transmission system infrastructure [13].

Adjusting the impedance and admittance of the transmission line is one method to control the power flow. FACTS devices, such as STATCOM, SVC, SSSC and UPFC, can be used to adjust the impedance and admittance of lines. These technologies can be inserted in series and/or shunt to perform control functions such as voltage regulation, system damping, and power flow control. The challenges that hindered the wide-scale deployment of FACTS devices are their high cost, maintenance complexity, and reliability concerns due to single source of failure [12]- 
[15], [22], [23]. To overcome these limitations the D-FACTS technology was introduced. The DSR was first proposed as a D-FACTS device [12], [14], [24].

As background for this paper, DSR concepts are reviewed here [16]. DSR technology is based on modifying the series reactance of a transmission line. DSR modules are mounted on the transmission conductor and may be activated to increase the series reactance of the line. When alternate flow paths exist in the transmission system the increase in series reactance of the line will cause flow to shift to the alternate paths.

The DSR addition affects the self-impedance of the line impedance matrix $\mathrm{Z}$ where

$$
\begin{aligned}
& Z_{i i}=\text { self-impedance of phase } \mathrm{i} \text {, and } \mathrm{i}=\mathrm{A}, \mathrm{B}, \mathrm{C} \text {. } \\
& \mathrm{Z}_{\mathrm{ij}}=\text { mutual impedance between phases } \mathrm{i} \text { and } \mathrm{j} \text {, and } \mathrm{i}, \mathrm{j}=\mathrm{A}, \mathrm{B}, \mathrm{C} \text {. }
\end{aligned}
$$

In the work here, the DSR adds reactance to the self-impedance of the line model. The value of the reactance added depends on the number of DSR modules activated and the selected reactance for each DSR module [16]-[17],[24]. In this study the DSR modules inject $50 \mu \mathrm{H} /$ module $(0.01885 \mathrm{ohms})$.

\section{Case Study Characteristics and Technology}

The IEEE 39 bus test system is modified to a 3-phase model and is used to study the deployment of DSRs for controlling power flow to alleviate overloads due to load growth. To address the consideration of generation in the design study, the ten generators in the IEEE standard model are replaced with three-phase equivalent voltage sources, where the solution of the model with the equivalent voltage sources is the same as the solution with the original power generators of the standard IEEE 39 bus model. In the load growth case studies that will be 
described in Section 5, it is assumed that the generators participate in picking up the load based upon the generation angle that exists for the base case solution. The way in which the generators are re-dispatched when the load grows will be shown in the examples. A different approach to the re-dispatch of the generators could have been used, which could influence the DSR design. However, the main purpose of this paper is to investigate DSR design for flow control given a generation dispatch scheme.

Thus, the system under study has ten voltage sources, 19 loads, and 35 lines. The transmission lines in the IEEE 39 bus standard transmission system are converted to $345 \mathrm{kV}$ lines modelled with the configuration and wiring shown in Fig. A of the Appendix. Line ratings and lengths are assumed as the IEEE 39 bus system does not provide them. Table A shows the assumed ratings and lengths used for the three phase lines of the system.

The authors have created 3-phase models of transmission systems for 7 different utilities in the U.S., where some of the transmission systems spanned multiple states, and included voltage levels of $230 \mathrm{kV}, 345 \mathrm{kV}$, and $500 \mathrm{kV}$, where the majority of the lines were not transposed. In several utilities there were no transposed lines in the transmission systems. The reasons for imbalance at the receiving end of a line can be due to impedance unbalance, load unbalance, and sending end voltage unbalance. This paper only addresses impedance unbalance, but as demonstrated in the case study in the paper, the effects of impedance unbalance (i.e., nontransposed lines) can be significant on flows and voltages. Thus, to investigate the effect on DSR design of the line impedance model, two cases are considered, an unbalanced, 3-phase impedance model, and a balanced, 3-phase impedance model. The balanced model is derived by assuming that the lines in the unbalanced model are transposed. In the unbalanced model the self-impedances are unequal with symmetrical but unequal off diagonal elements. The same is 
true for the shunt admittance matrix of the unbalanced model. In the balanced model the impedance matrix has diagonal elements that are equal in value and off diagonal elements that are zero [23], [25], [26].

For the unbalanced impedance model, the impedance matrix for the transmission lines is

$$
\mathrm{Z}_{\text {line }}=\left[\begin{array}{lll}
0.18+j 1.27 & 0.13+j 0.53 & 0.14+j 0.44 \\
0.13+j 0.53 & 0.19+j 1.26 & 0.14+j 0.52 \\
0.14+j 0.44 & 0.14+j 0.52 & 0.21+j 1.24
\end{array}\right] \quad \Omega / \text { mile (1) }
$$

and the shunt admittance matrix is

$$
\mathrm{Y}_{\text {line }}=\left[\begin{array}{ccc}
j 4.938 & -j 0.930 & -j 0.405 \\
-j 0.930 & j 4.977 & -j 0.941 \\
-j 0.405 & -j 0.941 & j 4.934
\end{array}\right] \mu \mathrm{S} / \text { mile (2) }
$$

The impedance matrix shown in (1) can be transformed into the sequence impedance matrix with the application of the symmetrical components transformation as given by

$\mathrm{Z}_{012}=[\mathrm{A}]^{-1} \cdot\left[\mathrm{Z}_{\text {line }}\right] \cdot[\mathrm{A}] \quad$ where: $\quad \mathrm{A}=\frac{1}{3}\left[\begin{array}{ccc}1 & 1 & 1 \\ 1 & a^{2} & a \\ 1 & a & a^{2}\end{array}\right] \& a=1 \underline{1120^{\circ}}(3)$

The resulting sequence impedance matrix is given by

$\mathrm{Z}_{012}=\left[\begin{array}{ccc}0.478+j 2.271 & -0.041-j 0.012 & 0.020+j 0.002 \\ 0.020+j 0.002 & 0.054+j 0.757 & 0.048+j 0.026 \\ -0.041-j 0.012 & -0.047+j 0.028 & 0.054+j 0.757\end{array}\right] \quad \Omega /$ mile (4)

The off-diagonal elements of the sequence impedance matrix are not zero, which implies that there is mutual coupling between sequences. With the off-diagonal elements nonzero, the three sequence networks representing the line will not be independent. However, it is observed that the off-diagonal elements are small relative to the diagonal terms. In high-voltage transmission lines it is usually assumed that the lines are transposed. The transposition can be simulated by replacing the diagonal terms of the impedance matrix with the average value of the diagonal 
terms $(0.19+\mathrm{j} 1.26)$, and replacing each off-diagonal term with the average of the off-diagonal terms $(0.14+\mathrm{j} 0.50)$.

$Z_{a b c}=\left[\begin{array}{lll}0.19+j 1.26 & 0.14+j 0.50 & 0.14+j 0.50 \\ 0.14+j 0.50 & 0.19+j 1.26 & 0.14+j 0.50 \\ 0.14+j 0.50 & 0.14+j 0.50 & 0.19+j 1.26\end{array}\right] \quad \Omega /$ mile (5)

Using this modified phase impedance matrix (5) in the symmetrical component transformation equation results in the modified sequence impedance matrix (6)

$\mathrm{Z}_{012}=\left[\begin{array}{ccc}0.478+\mathrm{j} 2.271 & 0 & 0 \\ 0 & 0.054+\mathrm{j} 0.757 & 0 \\ 0 & 0 & 0.054+\mathrm{j} 0.757\end{array}\right] \Omega /$ mile (6)

Now the off-diagonal terms are equal to zero, meaning there is no mutual coupling between sequence networks, and the three sequence networks representing the line are independent. The positive sequence admittance matrix can be accordingly obtained.

Thus, for the balanced model, which is the positive sequence representation of the transmission line, the impedance matrix and shunt admittance matrix for the transmission lines are represented in (7) and (8), respectively:

$$
\begin{aligned}
& \mathrm{Z}_{+\mathrm{ve}}=\left[\begin{array}{ccc}
0.054+j 0.75 & 0 & 0 \\
0 & 0.054+j 0.75 & 0 \\
0 & 0 & 0.054+j 0.75
\end{array}\right] \Omega / \text { mile (7) } \\
& \mathrm{Y}_{+\mathrm{ve}}=\left[\begin{array}{ccc}
j 0.0058 & 0 & 0 \\
0 & j 0.0058 & 0 \\
0 & 0 & j 0.0058
\end{array}\right] \mu \mathrm{S} / \mathrm{mile}(8)
\end{aligned}
$$

Concerning the practical deployment of DSRs, the authors considered deploying a maximum of 24 DSRs across the 3 phases per tower, where there are 4 DSRs per phase on each side of the tower. As the tower span for a $345 \mathrm{kV}$ line can have 10 towers per mile [27], 240 DSRs (24 DSRs x 10 towers) can be placed per mile on the 3 phases. 
The motivation to study the behavior of the DSRs with different impedance models is that the transmission lines in many power systems are not transposed and as a result have unbalanced immittances [18]-[21]. One aim of this study is to investigate the differences in DSR design results that occur between using the balanced immittance model and using the unbalanced immittance model.

\section{DSR Design Algorithm}

The DSR design and placement algorithm is presented in this section. The DSR design here assumes the deployment of the same number of DSRs per phase. DSRs are deployed on the transmission lines that provide the largest MW flow decrease in the overloaded lines. This is accomplished by calculating for a given line the change in MW in the overloaded lines for DSR addition to the given line. Thus, a line receiving DSRs may not be overloaded. This sensitivity is dependent on the utilization factor of the line. Accordingly, a set of lines are selected for DSR placement. DSR modules are placed iteratively with a certain step size that is chosen as the number of modules per phase. The step size (i.e., the number of DSRs added per iteration, is an input to the program). In the work here 25 DSRs per step are used.Using the chosen step size and sensitivity results derived from a three-phase, unbalanced power flow, the DSRs are deployed iteratively until the stopping criteria is fulfilled [28]. The stopping criteria adopted is to have no overloads in the system. It should be noted that the term "deployed" as used in this paper means the number of DSRs that are installed. The number of DSRs that are "turned on" depends upon the loading situation. Fig. 1 is a flowchart that depicts the design algorithm for DSR deployment.

The following describes how lines are selected for DSR additions.

Let $\mathrm{O}_{\mathrm{Bij}}=$ overload of line $\mathrm{i}$ before DSRs are added to line $\mathrm{j}$, in MW $\mathrm{O}_{\text {Aij }}=$ overload of line $\mathrm{i}$ after DSRs are added to line $\mathrm{j}$, in MW 
Define

$$
\Delta \mathrm{OL}_{\mathrm{j}}=\Sigma \mathrm{O}_{\mathrm{Bij}}-\mathrm{O}_{\mathrm{Aij}}
$$

Thus $\Delta \mathrm{OL}_{\mathrm{j}}$ represents the total decrease in overloads considering all overloaded lines in the system where DSRs are added to line $\mathrm{j}$. The line 's' to add DSRs to is then selected by

$$
\Delta \mathrm{OL}_{\mathrm{s}}=\max \text { over } \mathrm{j}\left\{\Delta \mathrm{OL}_{\mathrm{j}}\right\}
$$

Thus, the line is selected where $\Delta \mathrm{OL}_{\mathrm{j}} / \mathrm{Dsr}$ is the greatest, where Dsr is the number of DSRs added at each step. The algorithm used is based upon Discrete Ascent Optimal Programming, which is a greedy algorithm [29]. The Discrete Ascent Optimal Programming algorithm uses the sensitivity of flow in one line due to the impedance change in another line. Since the sensitivities are calculated for each phase, the unbalanced effects are included. However, a symmetrical placement of DSRs is still used in this work.

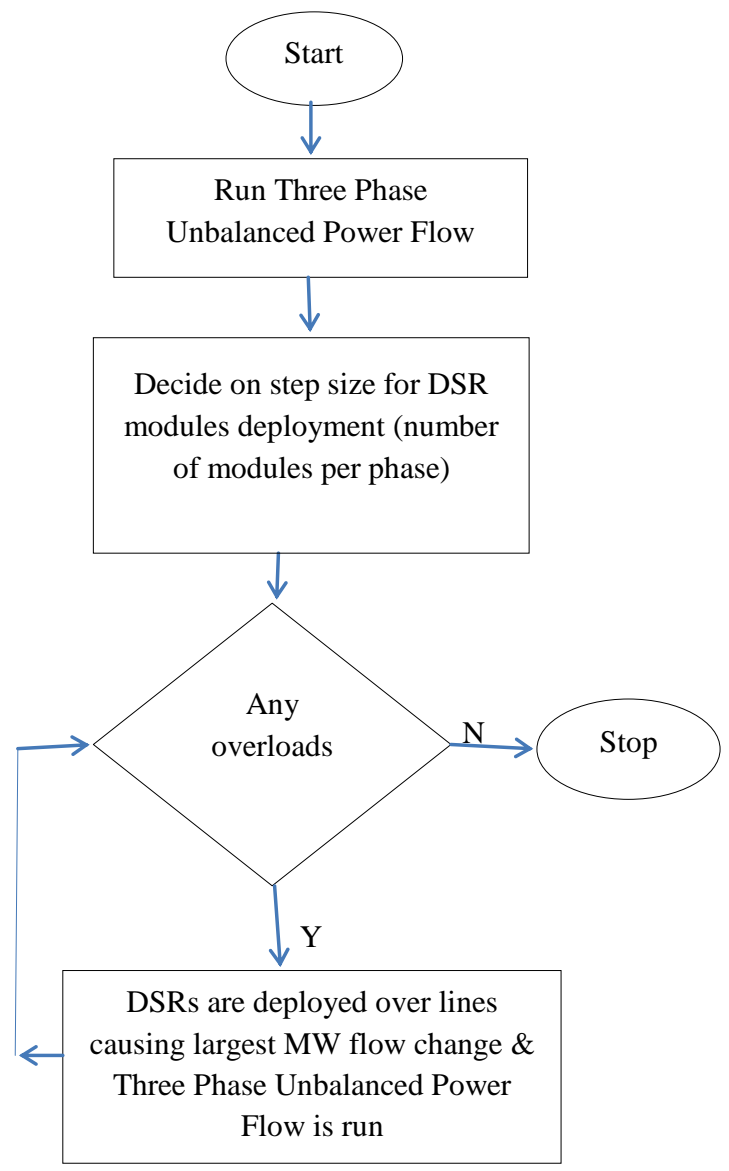


Fig.1 DSR deployment flow chart

\section{DSR Design Results for Load Growth}

DSRs can be controlled to increase the power transfer capacity of a meshed network. In this section this control is investigated using a series of load growth values for the two three-phase models described in Section 3, the balanced model and the unbalanced model.

The load is uniformly grown in $2 \%$ increments in both three-phase models until overloads are observed. When an overload is observed the DSR placement algorithm is used to try and find a DSR design that can be used to alleviate the overload. Fig.2 compares the total number of DSRs deployed for different system loads for the two models.

Table 1 shows the values of different total system loads adopted in the study as percentage of the base case and the corresponding MW values. Table 2 shows how the flows from the generators increase as the load is grown from $100 \%$ to $149 \%$. It also presents the $\%$ change in generation for each generator. Table 2 shows how all generators share in picking up the load.

Table 1

System load levels studied as percentage of base case and corresponding MWs

\begin{tabular}{|c|c|c|c|c|c|}
\cline { 2 - 6 } \multicolumn{1}{c|}{} & \multicolumn{5}{c|}{ System Load Levels Studied } \\
\hline Percentage & $141 \%$ & $143 \%$ & $145 \%$ & $147 \%$ & $149 \%$ \\
& & & & & \\
\hline MW & 8,670 & 8,793 & 8,917 & 9,040 & 9,163 \\
\hline
\end{tabular}

Table 2

Source voltages and generation for 100\% load and 149\% load of the three phases

\begin{tabular}{|c|c|c|c|c|c|c|}
\hline \multirow{2}{*}{\multicolumn{2}{|c|}{$\begin{array}{c}\text { Voltage Source } \\
\text { Bus } \\
\text { ( Fig. 3) }\end{array}$}} & \multicolumn{2}{|c|}{ Voltage } & \multirow{3}{*}{$\begin{array}{c}\text { Power Generated at } \\
\mathbf{1 0 0 \%}(\mathbf{M W}) \\
102.4\end{array}$} & \multirow{3}{*}{$\begin{array}{c}\text { Power Generated at } \\
\text { 149\% (MW) } \\
196.9\end{array}$} & \multirow{3}{*}{$\begin{array}{c}\text { \% Change in MW } \\
92.2\end{array}$} \\
\hline & & \multirow{2}{*}{\begin{tabular}{r|}
$\mathbf{k V}$ \\
13.3 \\
\end{tabular}} & \multirow{2}{*}{\begin{tabular}{|c|} 
Deg. \\
-4.58 \\
\end{tabular}} & & & \\
\hline 30 & $\mathbf{A}$ & & & & & \\
\hline & B & 13.3 & -124.5 & 98.96 & 184.9 & 86.8 \\
\hline & $\mathrm{C}$ & 13.3 & 115.42 & 102.5 & 189.5 & 84.8 \\
\hline
\end{tabular}




\begin{tabular}{|c|c|c|c|c|c|c|}
\hline \multirow[t]{3}{*}{32} & $\mathbf{A}$ & 12.4 & 1.6 & 182.5 & 246.7 & 35.1 \\
\hline & B & 12.4 & -118.4 & 185.8 & 242.9 & 30.7 \\
\hline & C & 12.4 & 121.6 & 186.3 & 246.2 & 32.1 \\
\hline \multirow[t]{3}{*}{33} & $\mathbf{A}$ & 12.6 & 2.07 & 199.8 & 300.8 & 50.5 \\
\hline & B & 12.6 & -117.9 & 202.1 & 297.4 & 47.1 \\
\hline & C & 12.6 & 122.07 & 202.0 & 297.0 & 47.0 \\
\hline \multirow[t]{3}{*}{34} & $\mathbf{A}$ & 12.8 & 0.63 & 177.5 & 278.3 & 56.7 \\
\hline & $\mathbf{B}$ & 12.8 & -119.3 & 175.7 & 272.7 & 55.2 \\
\hline & $\mathbf{C}$ & 12.8 & 120.6 & 178.5 & 277.0 & 55.1 \\
\hline \multirow[t]{3}{*}{35} & $\mathbf{A}$ & 13.3 & 4.04 & 193.7 & 292.3 & 50.9 \\
\hline & B & 13.3 & -115.9 & 198.2 & 290.0 & 46.3 \\
\hline & $\mathbf{C}$ & 13.3 & 124.04 & 196.5 & 288.8 & 46.9 \\
\hline \multirow[t]{3}{*}{36} & $\mathbf{A}$ & 13.5 & 6.73 & 184.4 & 252.2 & 36.7 \\
\hline & $\mathbf{B}$ & 13.5 & -113.2 & 186.6 & 249.6 & 33.7 \\
\hline & $\mathbf{C}$ & 13.5 & 126.73 & 186.8 & 250.9 & 34.3 \\
\hline \multirow[t]{3}{*}{37} & $\mathbf{A}$ & 13.0 & 1.15 & 174.6 & 247.6 & 41.8 \\
\hline & $\mathbf{B}$ & 13.0 & -118.8 & 175.8 & 243.5 & 38.5 \\
\hline & C & 13.0 & 121.15 & 177.5 & 246.5 & 38.8 \\
\hline \multirow[t]{3}{*}{38} & $\mathbf{A}$ & 13.0 & 6.44 & 272.9 & 384.3 & 40.8 \\
\hline & $\mathbf{B}$ & 13.0 & -113.5 & 279.6 & 385.6 & 37.9 \\
\hline & C & 13.0 & 126.44 & 279.5 & 386.5 & 38.2 \\
\hline \multirow[t]{3}{*}{39} & $\mathbf{A}$ & 13.0 & -11.11 & 364.5 & 646.0 & 77.2 \\
\hline & B & 13.0 & -131.1 & 359.6 & 633.1 & 76.0 \\
\hline & C & 13.0 & 108.89 & 371.2 & 643.0 & 73.2 \\
\hline \multirow[t]{3}{*}{ Slack } & $\mathbf{A}$ & 12.7 & 0 & $\begin{array}{l}199.9 \\
\end{array}$ & 270.2 & 35.1 \\
\hline & B & 12.7 & -120 & \begin{tabular}{|l|l|}
198.1 \\
\end{tabular} & 262.3 & 32.4 \\
\hline & C & 12.7 & 120 & 202.9 & 267.5 & 31.8 \\
\hline
\end{tabular}

For the load growth study, the study stopped at $149 \%$ load growth. As will be shown by the end of this section, at this load level the change in MW flow per DSR added becomes very small for both models.

It may be noted from Fig.2 that overloads start to occur in the balanced model at $141 \%$ load growth, whereas overloads do not occur in the unbalanced model until $145 \%$ load growth is reached. At the $141 \%$ and $143 \%$ load levels, the unbalanced model has no overloads and no DSRs are needed. However, the balanced model experiences overloads at the $141 \%$ and $143 \%$ load levels, where 75 DSRs and 900 DSRs are required, respectively. This is quite a significant difference between the 2 models.

The overload difference between the balanced and unbalanced model is due to the approximate impedances used in the balanced model. The assumed balanced model is conservative in predicting the overload before it actually would occur. It will also be shown in Fig. 2 that the 
balanced model is also conservative in predicting the number of DSRs that are required to eliminate overloads, and again this is due to the approximate impedances used in the assumed balanced model.

It should be noted that for the same loading condition the number of DSRs deployed using the balanced model is much higher than the number of DSRs deployed using the unbalanced model.

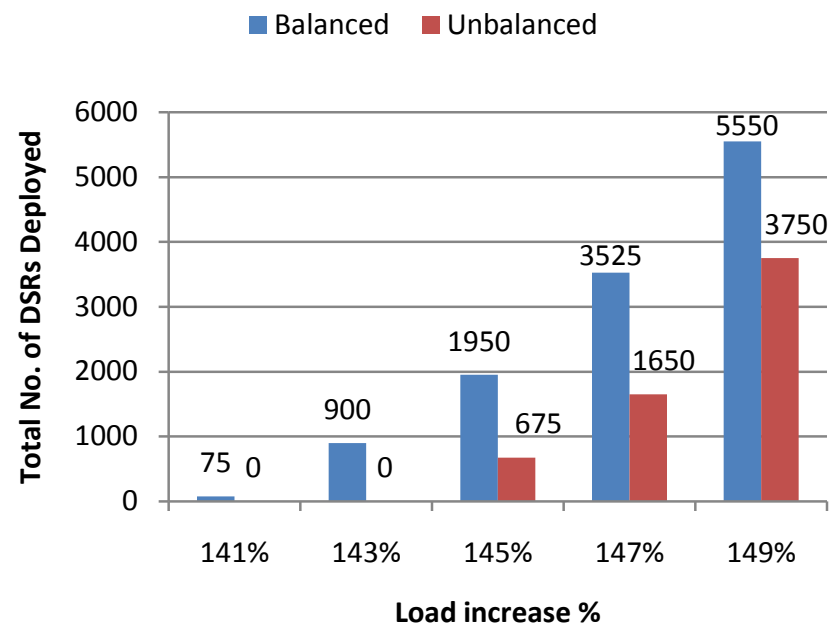

Fig. 2 Total number of DSRs deployed for balanced and unbalanced models as a function of system load

At the $149 \%$ load level, 3,750 DSRs are deployed in the unbalanced model to alleviate overloads whereas in the balanced model 5,550 DSRs are deployed. Using the balanced model thus results in an extremely conservative design. At the $149 \%$ load level the number of DSRs deployed on each line in the unbalanced model is shown in Fig. 3. Note that the crosses (x) that appear on some of the lines in Fig.3 indicate independent loop markers and are used by the power flow algorithm [28]. The independent loop markers correspond to the edges in the graph for the model that make up the complementary tree, or cotree.

Table 3 compares the designs for the two different models, and shows the discrepancy between the model predictions. 
Table 3

Comparisons of DSR design results between balanced and unbalanced models

\begin{tabular}{|c|c|c|c|c|c|c|c|c|c|c|}
\hline & \multicolumn{10}{|c|}{ Different System Loadings } \\
\hline & \multicolumn{2}{|c|}{$141 \%$} & \multicolumn{2}{|c|}{$143 \%$} & \multicolumn{2}{|r|}{$145 \%$} & \multicolumn{2}{|c|}{$147 \%$} & \multicolumn{2}{|c|}{$149 \%$} \\
\hline & $\begin{array}{c}\# \\
\text { DSR }\end{array}$ & $\begin{array}{c}\text { Slope } \\
\Delta \mathrm{MW} / \mathrm{DSR}\end{array}$ & \# DSR & $\begin{array}{c}\text { Slope } \\
\Delta \mathrm{MW} / \mathrm{DSR}\end{array}$ & $\begin{array}{c}\# \\
\text { DSR }\end{array}$ & $\begin{array}{c}\text { Slope } \\
\Delta \mathrm{MW} / \mathrm{DSR}\end{array}$ & $\begin{array}{c}\# \\
\text { DSR }\end{array}$ & $\begin{array}{c}\text { Slope } \\
\Delta \text { MW/DSR }\end{array}$ & $\begin{array}{c}\# \\
\text { DSR }\end{array}$ & $\begin{array}{c}\text { Slope } \\
\Delta \mathrm{MW} / \mathrm{DSR}\end{array}$ \\
\hline Balanced & 75 & 33.6 & 900 & 2.93 & 1950 & 1.41 & 3525 & 0.81 & 5550 & 0.54 \\
\hline Unbalanced & - & - & - & - & 675 & 4.09 & 1650 & 1.75 & 3750 & 0.80 \\
\hline Error \% & 100 & 100 & 100 & 100 & 65.38 & 65.38 & 53.19 & 53.19 & 48 & 32.43 \\
\hline
\end{tabular}

Fig.4 plots $\triangle \mathrm{MW} / \mathrm{DSR}$ as a function of system loading for both models, where $\Delta \mathrm{MW}$ represents the incremental load served when 1 DSR module is deployed at each load level.

Above the $145 \%$ load level, the $\Delta \mathrm{MW}$ /DSR slope has a smaller value for the assumed balanced model, which indicates that more DSRs will be required in the balanced model to affect a given change in flow. 
@- Equivalent Source

Line with DSR

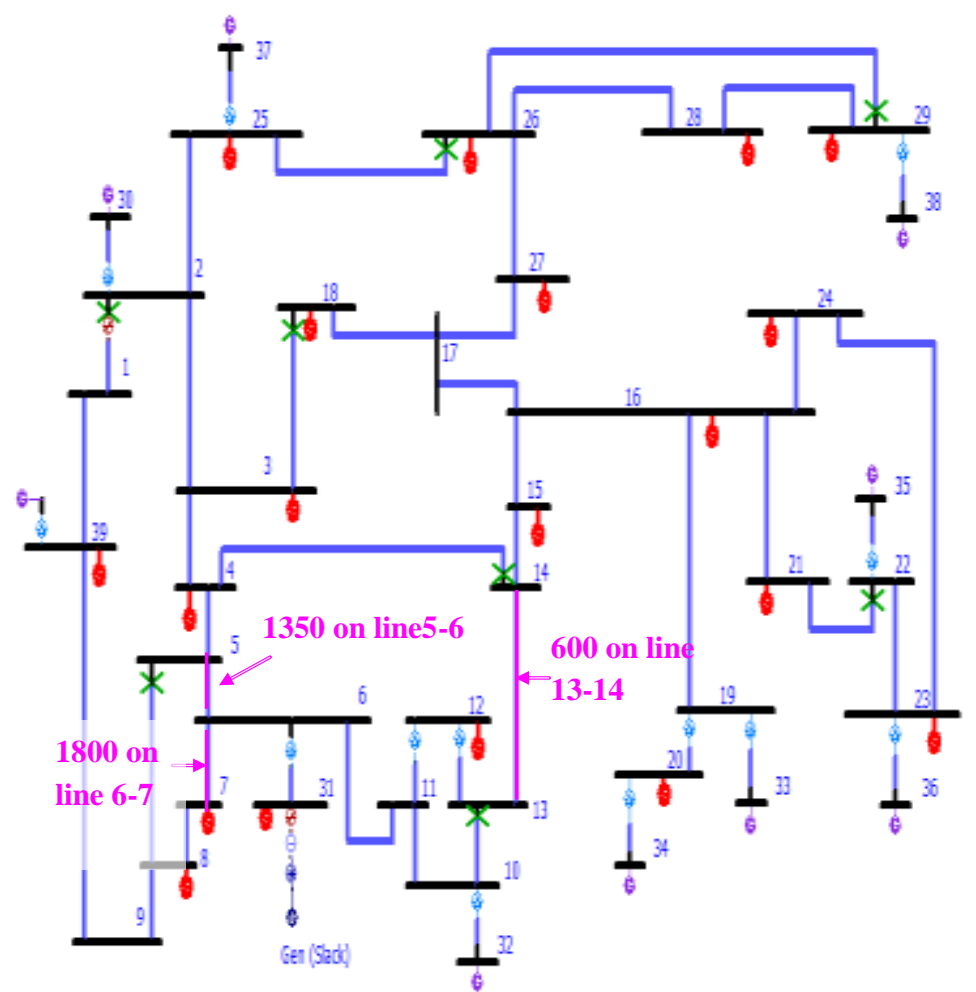

Fig. 3 DSRs deployed on the unbalanced model for a load growth of $149 \%$

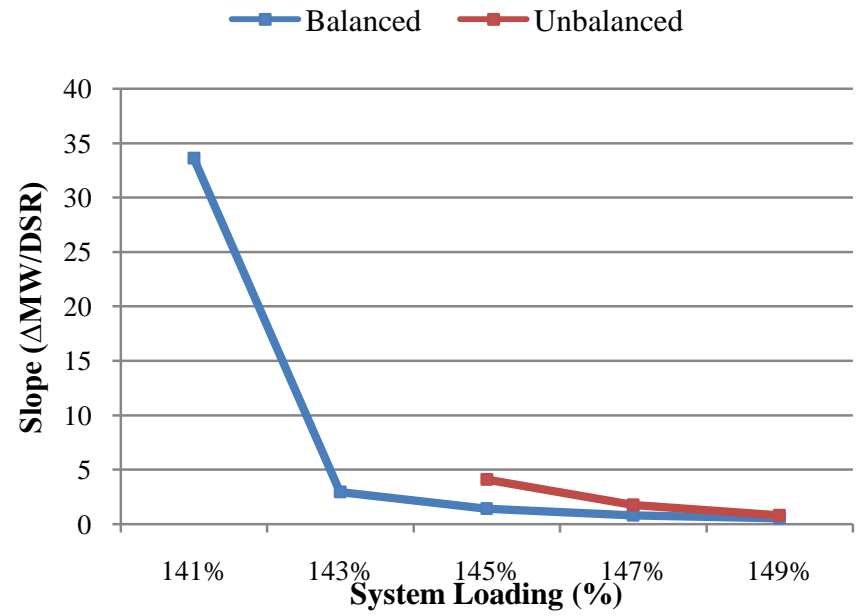

Fig. 4 MW change per DSR deployed for different system loadings for balanced and unbalanced models

\section{Conclusions}

This paper studied controlling power flow using Distributed Series Reactors (DSRs) and the effect that line models can have on the design. The IEEE 39 bus standard transmission system model was converted to a three-phase model using $345 \mathrm{kV}$ unbalanced line models and the 
generators were converted to three-phase equivalent voltage sources. A second model of the system was created by assuming that the lines in the unbalanced model are transposed. This model was referred to as the balanced model. The balanced model is the positive sequence representation of the system obtained using the symmetrical components transformation.

For a given loading, use of the balanced model in the design results in many more DSRs for control than when the unbalanced model is used in the design. It was shown that the balanced model assumption, if used when lines are truly unbalanced, can lead to significant errors. Since the majority of the transmission system in the U.S. is unbalanced, and since significant errors can arise when just the impedance unbalance is considered, as shown here, the authors believe that 3phase analysis of the transmission system should be pursued.

The application of DSRs can increase the utilization of the line capacity in a system. This increased utilization can delay or eliminate the need for building new transmission lines. Whether DSRs should be used to increase line utilization or new lines should be built is partly an economic question.

\section{Appendix}

The lattice tower structure for the $345 \mathrm{kV}$ line used to convert the IEEE 39 bus standard test system to a physical system is shown in Fig. A (a). An ACSR conductor with a 1.737 inches diameter and the stranding as $84 / 19$ is used with this tower structure [27]. Each phase has a bundled conductor having two ACSR conductors. The representation of each conductor using the equivalent GMR of the bundled conductors with the spacing in feet is shown in Fig. A (b).

Table A shows the ratings and lengths assigned for the three phase lines of the system. In converting from the per unit model, the length of the line with the lowest pu impedance was set to 20 miles, and the length of the line with the highest pu impedance was set to 100 miles, and 
then the lengths of all other lines was selected proportionally. As mentioned earlier in Section 3, the line ratings are assumed.

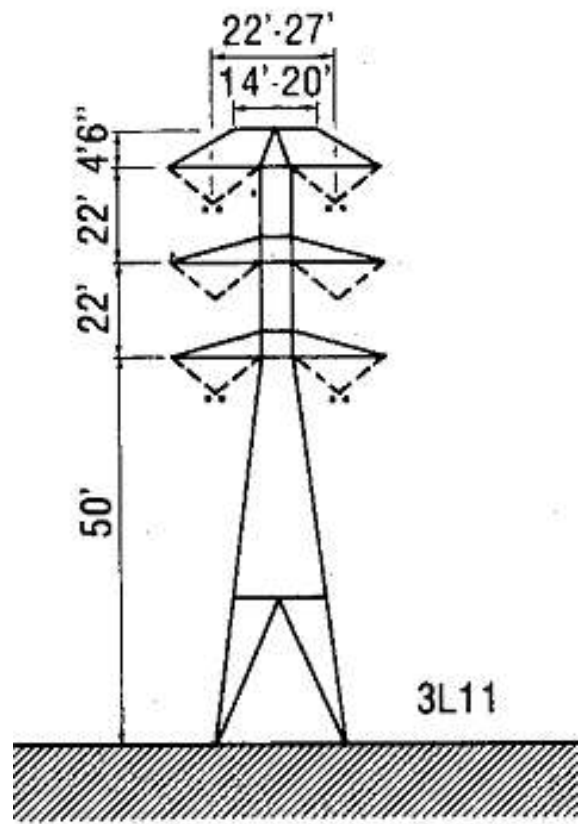

(a)

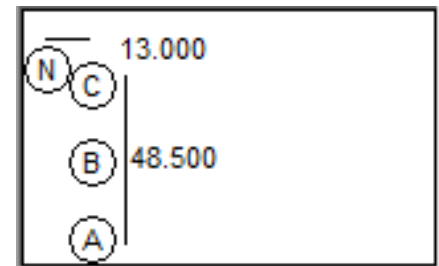

(b)

Fig. A. The 3-phase 345kV line (a) Lattice tower structure [27] (b) Equivalent representation of bundled conductors TableA

IEEE 39 bus three phase lines ratings and length

\begin{tabular}{|c|c|c|c|}
\hline From Bus & To Bus & $\begin{array}{c}\text { Line Rating } \\
\text { (MVA) }\end{array}$ & Length (miles) \\
\hline 1 & 2 & 400 & 71.39 \\
\hline 2 & 3 & 850 & 36.69 \\
\hline 2 & 25 & 1100 & 31.28 \\
\hline 3 & 18 & 400 & 34.28 \\
\hline 3 & 4 & 600 & 47.3 \\
\hline 4 & 14 & 600 & 33.72 \\
\hline 4 & 5 & 600 & 33.59 \\
\hline 5 & 8 & 600 & 31.47 \\
\hline 5 & 6 & 600 & 20 \\
\hline 6 & 11 & 600 & 27.48 \\
\hline
\end{tabular}




\begin{tabular}{|c|c|c|c|}
\hline 6 & 7 & 600 & 28.79 \\
\hline 7 & 8 & 600 & 22.67 \\
\hline 8 & 9 & 600 & 64.91 \\
\hline 10 & 13 & 600 & 22.28 \\
\hline 10 & 11 & 600 & 22.28 \\
\hline 13 & 14 & 500 & 30.02 \\
\hline 14 & 15 & 600 & 45.49 \\
\hline 15 & 16 & 750 & 29.09 \\
\hline 16 & 24 & 500 & 24.39 \\
\hline 16 & 21 & 1000 & 34.52 \\
\hline 16 & 19 & 1000 & 42.55 \\
\hline 16 & 17 & 600 & 28.41 \\
\hline 17 & 27 & 500 & 39.61 \\
\hline 17 & 18 & 600 & 27.48 \\
\hline 19 & 20 & 500 & 34.91 \\
\hline 21 & 22 & 1200 & 35.18 \\
\hline 22 & 23 & 500 & 29.32 \\
\hline 23 & 24 & 1200 & 63.17 \\
\hline 25 & 26 & 600 & 59.7 \\
\hline 26 & 29 & 1100 & 100 \\
\hline 26 & 28 & 1100 & 79.83 \\
\hline 26 & 27 & 1100 & 36.17 \\
\hline 28 & 29 & 1100 & 36.7 \\
\hline 39 & 1 & 500 & 49.81 \\
\hline 39 & 9 & 600 & 49.81 \\
\hline
\end{tabular}

\section{Acknowledgment}

This research has been sponsored by the DOE ARPA-E GENI program, Smart Wire Grid, Electrical Distribution Design, and the Virginia Tech Middle East and North Africa (VT-MENA). 


\section{References}

[1] "Transmission Projects: At A Glance," Edison Electric Institute, March 2013.

[2] M. S. Sarma, J. D. Glover and T. J. Overbye, Power System Analysis and Design, $5^{\text {th }}$ ed., SI, Global Engineering, 2008-2012.

[3] J. Samuelsson, A. Wilk-Wilczynski and J. Nizovoy, "Increase of transmission capacity by resource pooling in Argentina," in Proc.IEEE PES SM, vol. 1, no. 38,42, 2001.

[4] M. Alam, M. Razzak, M. Hasan and A. Chowdhury, "Transmission capacity enhancement of EastWest Interconnectors using series-shunt compensation," in Proc. 7th ICECE, pp. 579,582, 20-22 Dec. 2012.

[5] H. Glavitsch and M. Rahmani, "Increased transmission capacity by forced symmetrization," IEEE Trans. Power Systems, vol. 13, no. 1, pp. 79,85, Feb 1998.

[6] T. Krontiris, A. Wasserrab and G. Balzer, "Weather-based loading of overhead lines Consideration of conductor's heat capacity," in Proc. MEPS, pp. 1,8, 20-22 Sept. 2010.

[7] S. C. Müller, U. Häger and C. Rehtanz, "A Multi-Agent System for Adaptive Power Flow Control in Electrical Transmission Systems," IEEE Trans. Industrial Informatics, 2013.

[8] H. Rafiq, S. Shakeel, . S. Nawaz, M. . K. Bashir, Y. Saleem, M. Saleem and T. Izhar, "Control System Design of UPFC for Optimal Power Flow Control," in Proc. ICOSST, 2013.

[9] S. T. Kalyani and G. T. Das, "Control and Performance of UPFC Connected To a Transmission Line," in Proc.IPEC, 3-6 Dec. 2007.

[10] K. Belacheheb and S. Saadate, "Compensation of the electrical mains by means of Unified Power Flow Controller (UPFC) - Comparison of three control methods," in Proc. ICHQP, 2000.

[11] D. J. Gotham and G. T. Heydt , "Power Flow Control And Power Flowstudies For Systems With Facts Devices," IEEE Trans. Power Systems, vol. 13, no. 1, pp. 60-65, February 1998.

[12] F. Kreikebaum, M. Imayavaramban and D. Divan, "Active smart wires: An inverter-less static series compensator," in Proc. ECCE, pp. 3626,3630, 12-16 Sept. 2010.

[13] D. Das, F. Kreikebaum, D. Divan and F. Lambert, "Reducing transmission investment to meet Renewable Portfolio Standards Using Smart Wires," in Proc. IEEE PES T\&D, pp. 1,7, 19-22 April 2010.

[14] D. Divan, W. Brumsickle, R. Schneider, B. Kranz, R. Gascoigne, D. Bradshaw, M. Ingram and I. Grant, "A Distributed Static Series Compensator System for Realizing Active Power Flow Control on Existing Power Lines," IEEE Trans. on Power Delivery, vol. 22, no. 1, pp. 642,649, Jan. 2007.

[15] D. Divan and H. Johal, "Distributed FACTS - A New Concept for Realizing Grid Power Flow Control," in Proc. IEEE PESC, June 2005.

[16] S. Omran, R. Broadwater, J. Hambrick andM. Dilek, "DSR Design Fundamentals: Power Flow Control," presented in 2014 IEEE PES General Meeting, July 2014.

[17] S. Omran, R. Broadwater, J. Hambrick, M. Dilek, C.Thomas, and F. Kreikebaum, "Power Flow Control and N-1 Contingency Analysis with DSRs in Unbalanced Transmission Networks,"Electric Power Systems Research-Elsevier, vol. 136, pp. 223-231, 2016.

[18] J. B. Rossman, T. L. Laughner, A. M. Murphy, D. E. Marler and G. L. Kobet, "Transmission voltage unbalance evaluation," Future of Instrumentation International Workshop (FIIW), 2012, Gatlinburg, TN, 2012, pp. 1-4. doi: 10.1109/FIIW.2012.6378338

[19] U. Jayatunga, S. Perera, P. Ciufo and A. P. Agalgaonkar, "Deterministic methodologies for the quantification of voltage unbalance propagation in radial and interconnected networks," in IET Generation, Transmission \& Distribution, vol. 9, no. 11, pp. 1069-1076, 862015. doi: 10.1049/iet-gtd.2014.0661

[20] North American Reliability Corporation "Power Plant and Transmission System Protection Coordination", NERC System Protection and Control Subcommittee, July 2010.

[21] IEC , "Limits - Assessment of emission limits for the connection of unbalanced installations to MV, HV and 
EHV power systems", February 2008.

[22] Massachusetts Institute of Technology, "The Future of The Electric Grid, An Interdisciplinary MIT Study," 2011.

[23] E. Hirst, "Do We Need More Transmission," Elsevier Science, The Electricity Journal, pp. 78-89, 2000.

[24] I. Grant, F. Kreikebaum, J. Shultz, S. Omran and R. Broadwater, "Initial Field Trials of Distributed Series Reactors and Implications for Future Application," presented in CIGRE US NCGFS, 2014.

[25] H. Saadat, Power System Analysis, WCB/McGraw-Hill, 1999, 1999.

[26] W. Kersting, Distribution System Modeling and Analysis, CRC Press, 2002.

[27] EPRI, Transmission Line Reference Book - 345kV and above, Electric Power Research Institute, Third Edition, 1982.

[28] M. Dilek, F. de Leon, R. Broadwater, S. Lee, "A Robust Multiphase Power Flow for General Distribution Networks," IEEE Trans. Power Systems, vol.25, no.2, pp.760,768, May 2010.

[29] R.Broadwater, P.Dolloff, T.Herdman, R.Karamikhova, A. Sargent "Minimum Loss Optimization in Distribution Systems: Discrete Ascent Optimal Programming,", Elec. Power Sys. Research Journal, vol. 36, no. 2, pp. 113-121, 1996. 